Bull. Korean Math. Soc. 50 (2013), No. 1, pp. 135-142

http://dx.doi.org/10.4134/BKMS.2013.50.1.135

\title{
THE RIGIDITY OF MINIMAL SUBMANIFOLDS IN A LOCALLY SYMMETRIC SPACE
}

\author{
Shunjuan CaO
}

\begin{abstract}
In the present paper, we discuss the rigidity phenomenon of closed minimal submanifolds in a locally symmetric Riemannian manifold with pinched sectional curvature. We show that if the sectional curvature of the submanifold is no less than an explicitly given constant, then either the submanifold is totally geodesic, or the ambient space is a sphere and the submanifold is isometric to a product of two spheres or the Veronese surface in $S^{4}$
\end{abstract}

\section{Introduction}

Let $M^{n}$ be an $n$-dimensional closed minimal submanifold in an $(n+p)$ dimensional Riemannian manifold $N^{n+p}$. We denote by $S$ the squared norm of the second fundamental form of $M$. If $N^{n+p}$ is the $(n+p)$-dimensional unit sphere $S^{n+p}$, a famous rigidity theorem due to Simons [11], Lawson [8] and Chern-do Carmo-Kobayashi [1] says that if $S \leq \frac{n}{2-1 / p}$, then either $M$ is totally geodesic, or $M$ is one of the Clifford minimal hypersurfaces $S^{k}\left(\sqrt{\frac{k}{n}}\right) \times$ $S^{n-k}\left(\sqrt{\frac{n-k}{n}}\right), k=1, \ldots, n-1$, or $n=2, p=2$, and $M$ is the Veronese surface in $S^{4}$. Further discussions have been carried out by many other authors $[2,9,12,13,14,15]$, etc.

Denote by $K_{M}$ the sectional curvature of $M$. In 1975, Yau [16] proved the following rigidity theorem.

Theorem 1.1 ([16]). Let $M^{n}$ be an n-dimensional oriented closed minimal submanifold in $S^{n+p}$. If $K_{M} \geq \frac{p-1}{2 p-1}$, then either $M$ is the totally geodesic sphere, the standard immersion of the product of two spheres, or $M$ is the Veronese surface in $S^{4}$.

Received June 3, 2011.

2010 Mathematics Subject Classification. 53C24, 53C40.

Key words and phrases. minimal submanifold, rigidity, sectional curvature, locally symmetric space. 
Later, Itoh [7] proved that if $K_{M} \geq \frac{n}{2(n+1)}$, then $M$ is the totally geodesic sphere, or the Veronese surface in $S^{4}$. Recently, Gu-Xu [5] made an improvement of Yau's rigidity theorem. They obtained the following theorem.

Theorem $1.2([5])$. Let $M^{n}$ be an $n$-dimensional oriented closed minimal submanifold in $S^{n+p}$. If $K_{M} \geq \frac{\operatorname{sgn}(p-1) p}{2(p+1)}$, then either $M$ is the totally geodesic sphere, the standard immersion of the product of two spheres, or $M$ is the Veronese surface in $S^{4}$. Here $\operatorname{sgn}(\cdot)$ is the standard sign function.

In this paper, we discuss the rigidity of minimal submanifolds in a Riemannian manifold. We assume that the ambient space is locally symmetric and $\delta$-pinched. We obtain the following theorem.

Theorem 1.3. Let $M^{n}$ be an n-dimensional oriented closed minimal submanifold in an $n$-dimensional simply connected and locally symmetric Riemannian manifold $N^{n+p}$. Suppose the sectional curvature $K_{N}$ of $N$ satisfies $\delta \leq K_{N} \leq 1$. If

$K_{M} \geq \frac{4}{3 n(p+1)}(n-1)^{\frac{1}{2}}(p-1)(p+2)(1-\delta)+\left(\frac{p+2}{2(p+1)}-\frac{\delta}{p+1}\right) \operatorname{sgn}(p-1)$,

then either $M$ is totally geodesic, or $N^{n+p}=S^{n+p}$ and $M$ is isometric to the standard immersion of the product of two spheres or the Veronese surface in $S^{4}$.

\section{Preliminaries}

Let $M^{n}$ be an $n$-dimensional closed minimal submanifold in an $(n+p)$ dimensional Riemannian manifold $N^{n+p}$. We shall make use of the following convention on the range of indices:

$$
1 \leq A, B, C, \ldots \leq n+p ; 1 \leq i, j, k, \ldots \leq n ; n+1 \leq \alpha, \beta, \gamma, \ldots \leq n+p .
$$

Choose a local field of orthonormal frames $\left\{e_{A}\right\}$ in $N$ such that, restricted to $M$, the $e_{i}$ 's are tangent to $M$. Let $\left\{\omega_{A}\right\}$ and $\left\{\omega_{A B}\right\}$ be the dual frame field and the connection 1-forms of $N$, respectively. Restricting these forms to $M$, we have

$$
\begin{aligned}
& \omega_{\alpha i}=\sum_{j} h_{i j}^{\alpha} \omega_{j}, h_{i j}^{\alpha}=h_{j i}^{\alpha}, \\
& h=\sum_{\alpha, i, j} h_{i j}^{\alpha} \omega_{i} \otimes \omega_{j} \otimes e_{\alpha}, \xi=\frac{1}{n} \sum_{\alpha, i} h_{i i}^{\alpha} e_{\alpha}, \\
& R_{i j k l}=\bar{R}_{i j k l}+\sum_{\alpha}\left(h_{i k}^{\alpha} h_{j l}^{\alpha}-h_{i l}^{\alpha} h_{j k}^{\alpha}\right), \\
& R_{\alpha \beta k l}=\bar{R}_{\alpha \beta k l}+\sum_{i}\left(h_{i k}^{\alpha} h_{i l}^{\beta}-h_{i l}^{\alpha} h_{i k}^{\beta}\right),
\end{aligned}
$$


where $h, \xi, R_{i j k l}, R_{\alpha \beta k l}$, and $\bar{R}_{A B C D}$ are the second fundamental form, the mean curvature vector, the curvature tensor, the normal curvature tensor of $M$, and the curvature tensor of $N$, respectively. We set

$$
S=|h|^{2}, H=|\xi|, H_{\alpha}=\left(h_{i j}^{\alpha}\right)_{n \times n} .
$$

Then $M$ is minimal if and only if $H=0$.

Denote the first and second covariant derivatives of $h_{i j}^{\alpha}$ by $h_{i j k}^{\alpha}$ and $h_{i j k l}^{\alpha}$, respectively. We have

$$
\begin{aligned}
& \sum_{k} h_{i j k}^{\alpha} \omega_{k}=d h_{i j}^{\alpha}-\sum_{k} h_{i k}^{\alpha} \omega_{k j}-\sum_{k} h_{k j}^{\alpha} \omega_{k i}-\sum_{\beta} h_{i j}^{\beta} \omega_{\beta \alpha} \\
& \sum_{l} h_{i j k l}^{\alpha} \omega_{l}=d h_{i j k}^{\alpha}-\sum_{l} h_{i j l}^{\alpha} \omega_{l k}-\sum_{l} h_{i l k}^{\alpha} \omega_{l j}-\sum_{l} h_{l j k}^{\alpha} \omega_{l i}-\sum_{\beta} h_{i j k}^{\beta} \omega_{\beta \alpha} .
\end{aligned}
$$

Then

$$
\begin{gathered}
h_{i j k}^{\alpha}-h_{i k j}^{\alpha}=-\bar{R}_{\alpha i j k}, \\
h_{i j k l}^{\alpha}-h_{i j l k}^{\alpha}=\sum_{m} h_{m i}^{\alpha} R_{m j k l}+h_{m j}^{\alpha} R_{m i k l}-\sum_{\beta} h_{i j}^{\beta} R_{\alpha \beta k l} .
\end{gathered}
$$

Consider $\bar{R}_{\alpha i j k}$ as a section of the bundle $N M \otimes T^{*} M \otimes T^{*} M \otimes T^{*} M$. Its covariant derivative $\bar{R}_{\alpha i j k l}$ is defined by

$$
\begin{aligned}
& \sum_{l} \bar{R}_{\alpha i j k l} \omega_{l} \\
= & d \bar{R}_{\alpha i j k}-\sum_{m} \bar{R}_{\alpha m j k} \omega_{m i}-\sum_{m} \bar{R}_{\alpha i m k} \omega_{m j}-\sum_{m} \bar{R}_{\alpha i j m} \omega_{m k}-\sum_{\beta} \bar{R}_{\beta i j k} \omega_{\beta \alpha} .
\end{aligned}
$$

Let $\bar{R}_{A B C D, E}$ be the covariant derivative of $\bar{R}_{A B C D}$ as a curvature tensor of $N$. Restricted to $M, \bar{R}_{\alpha i j k, l}$ is given by

$\bar{R}_{\alpha i j k, l}=\bar{R}_{\alpha i j k l}-\sum_{\beta} \bar{R}_{\alpha \beta j k} h_{i l}^{\beta}-\sum_{\beta} \bar{R}_{\alpha i \beta k} h_{j l}^{\beta}-\sum_{\beta} \bar{R}_{\alpha i j \beta} h_{k l}^{\beta}+\sum_{m} \bar{R}_{m i j k} h_{m l}^{\alpha}$.

Now we assume that the ambient space $N$ is locally symmetric, i.e., $\bar{R}_{A B C D, E}$ $=0$. Then we have

(5) $\quad \bar{R}_{\alpha i j k l}=\sum_{\beta} \bar{R}_{\alpha \beta j k} h_{i l}^{\beta}+\sum_{\beta} \bar{R}_{\alpha i \beta k} h_{j l}^{\beta}+\sum_{\beta} \bar{R}_{\alpha i j \beta} h_{k l}^{\beta}-\sum_{m} \bar{R}_{m i j k} h_{m l}^{\alpha}$.

The Laplacian $\triangle h_{i j}^{\alpha}$ of $h$ is defined by $\triangle h_{i j}^{\alpha}=\sum_{k} \triangle h_{i j k k}^{\alpha}$. Then from (1), (4), (5) and the minimality of $M$, we have for any real number $a$,

$$
\begin{aligned}
\sum_{i, j, \alpha} h_{i j}^{\alpha} \triangle h_{i j}^{\alpha}= & 4 \sum_{\alpha, \beta} \sum_{i, j, k} h_{j k}^{\alpha} h_{i l}^{\beta} \bar{R}_{\alpha \beta i j}-\sum_{\alpha, \beta} \sum_{i, j, k} h_{i j}^{\alpha} h_{i j}^{\beta} \bar{R}_{\alpha k \beta k} \\
& +(1-a) \sum_{\alpha} \sum_{i, j, k, m} h_{i j}^{\alpha}\left(h_{m k}^{\alpha} \bar{R}_{m i j k}+h_{m i}^{\alpha} \bar{R}_{m k j k}\right)
\end{aligned}
$$




$$
\begin{aligned}
& +a \sum_{\alpha, \beta}\left[\operatorname{tr}\left(H_{\alpha} H_{\beta}\right)\right]^{2} \\
& +(1+a) \sum_{\alpha} \sum_{i, j, k, m} h_{i j}^{\alpha}\left(h_{m k}^{\alpha} R_{m i j k}+h_{m i}^{\alpha} R_{m k j k}\right) \\
& -\sum_{\alpha, \beta}\left[\operatorname{tr}\left(H_{\alpha}^{2} H_{\beta}^{2}\right)-\operatorname{tr}\left(H_{\alpha} H_{\beta}\right)^{2}\right] .
\end{aligned}
$$

The DDVV inequality proved by $\mathrm{Lu}[10]$, Ge and Tang [3] is stated as follows.

Lemma 2.1 (DDVV Inequality). Let $B_{1}, \ldots, B_{m}$ be symmetric $(n \times n)$ real matrices. Then

$$
\sum_{r, s=1}^{m}\left\|\left[B_{r}, B_{s}\right]\right\|^{2} \leq\left(\sum_{r=1}^{m}\left\|B_{r}\right\|^{2}\right)^{2}
$$

where the equality holds if and only if under some rotation all $B_{r}$ 's are zero except two matrices which can be written as

$$
\tilde{B}_{r}=P\left(\begin{array}{ccccc}
0 & \mu & 0 & \cdots & 0 \\
\mu & 0 & 0 & \cdots & 0 \\
0 & 0 & 0 & \cdots & 0 \\
\vdots & \vdots & \vdots & \ddots & \vdots \\
0 & 0 & 0 & \cdots & 0
\end{array}\right) P^{t}, \quad \tilde{B}_{s}=P\left(\begin{array}{ccccc}
\mu & 0 & 0 & \cdots & 0 \\
0 & -\mu & 0 & \cdots & 0 \\
0 & 0 & 0 & \cdots & 0 \\
\vdots & \vdots & \vdots & \ddots & \vdots \\
0 & 0 & 0 & \cdots & 0
\end{array}\right) P^{t}
$$

where $P$ is an orthogonal $(n \times n)$-matrix. Here $\|\cdot\|^{2}$ denotes the sum of squares of entries of the matrix and $[A, B]=A B-B A$ is the commutator of the matrices $A, B$.

In the proof of the theorem, we also use the following Berger's inequalities.

Lemma $2.2([4])$. Let $N$ be an $(n+p)$-dimensional Riemannian manifold satisfying $a \leq K_{N} \leq b$. Let $\left\{e_{A}\right\}$ be a local orthonormal basis. Then

(1) $\bar{R}_{A B C D} \leq \frac{2}{3}(b-a)$ for all distinct $A, B, C, D$.

(2) $\bar{R}_{A C B C} \leq \frac{1}{2}(b-a)$ for $A \neq B$.

\section{Proof of the main theorem}

To prove Theorem 1.3, we first give some estimates.

For a fixed $\alpha$, we choose $\left\{e_{i}\right\}$ such that $h_{i j}^{\alpha}=\lambda_{i}^{\alpha} \delta_{i j}$. Then

$$
\begin{aligned}
& 4 \sum_{\beta} \sum_{i, j, k} h_{j k}^{\alpha} h_{i l}^{\beta} \bar{R}_{\alpha \beta i j} \\
= & 4 \sum_{\beta \neq \alpha} \sum_{i \neq k} \lambda_{k}^{\alpha} h_{i k}^{\beta} \bar{R}_{\alpha \beta i k} \\
\geq & -\frac{8}{3}(1-\delta) \sum_{\beta \neq \alpha} \sum_{i \neq k}\left|\lambda_{k}^{\alpha} h_{i k}^{\beta}\right|
\end{aligned}
$$




$$
\begin{aligned}
& \geq-\frac{4}{3}(1-\delta) \sum_{\beta \neq \alpha} \sum_{i \neq k}\left[(n-1)^{-\frac{1}{2}}\left(\lambda_{k}^{\alpha}\right)^{2}+(n-1)^{\frac{1}{2}}\left(h_{i k}^{\beta}\right)^{2}\right] \\
& =-\frac{4}{3}(1-\delta)(n-1)^{\frac{1}{2}}(p-1) \operatorname{tr} H_{\alpha}^{2}-\frac{4}{3}(1-\delta)(n-1)^{\frac{1}{2}} \sum_{\beta \neq \alpha} \operatorname{tr} H_{\beta}^{2} .
\end{aligned}
$$

Then we have

$$
4 \sum_{\alpha, \beta} \sum_{i, j, k} h_{j k}^{\alpha} h_{i l}^{\beta} \bar{R}_{\alpha \beta i j} \geq-\frac{8}{3}(1-\delta)(n-1)^{\frac{1}{2}}(p-1) S .
$$

Since $\left(\operatorname{tr}\left(H_{\alpha} H_{\beta}\right)\right)$ is a symmetric $(p \times p)$-matrix, we can choose the normal frame fields $\left\{e_{\alpha}\right\}$ such that

$$
\operatorname{tr}\left(H_{\alpha} H_{\beta}\right)=\operatorname{tr} H_{\alpha}^{2} \cdot \delta_{\alpha \beta} .
$$

Then

$$
\begin{aligned}
-\sum_{\alpha, \beta} \sum_{i, j, k} h_{i j}^{\alpha} h_{i j}^{\beta} \bar{R}_{\alpha k \beta k} & =-\sum_{\alpha, \beta} \operatorname{tr}\left(H_{\alpha} H_{\beta}\right) \sum_{k} \bar{R}_{\alpha k \beta k} \\
& =-\sum_{\alpha} \operatorname{tr}\left(H_{\alpha}^{2}\right) \sum_{k} \bar{R}_{\alpha k \alpha k} \\
& \geq-n S .
\end{aligned}
$$

We also have

$$
\begin{aligned}
\sum_{i, j, k, m} h_{i j}^{\alpha}\left(h_{m k}^{\alpha} \bar{R}_{m i j k}+h_{m i}^{\alpha} \bar{R}_{m k j k}\right) & =\frac{1}{2} \sum_{i, k}\left(\lambda_{i}^{\alpha}-\lambda_{k}^{\alpha}\right)^{2} \bar{R}_{i k i k} \\
& \geq \frac{1}{2} \delta \sum_{i, k}\left(\lambda_{i}^{\alpha}-\lambda_{k}^{\alpha}\right)^{2} \\
& =n \delta \operatorname{tr}\left(H_{\alpha}^{2}\right) .
\end{aligned}
$$

Hence

(9)

$$
\sum_{\alpha} \sum_{i, j, k, m} h_{i j}^{\alpha}\left(h_{m k}^{\alpha} \bar{R}_{m i j k}+h_{m i}^{\alpha} \bar{R}_{m k j k}\right) \geq n \delta S .
$$

Similarly, we have

$$
\sum_{\alpha} \sum_{i, j, k, m} h_{i j}^{\alpha}\left(h_{m k}^{\alpha} R_{m i j k}+h_{m i}^{\alpha} R_{m k j k}\right) \geq n K_{\min } S,
$$

where $K_{\text {min }}$ is the minimum of the sectional curvature at a point.

On the other hand, by a direct computation and the DDVV inequality, we obtain

$$
\begin{aligned}
\sum_{\alpha, \beta} \operatorname{tr}\left(H_{\alpha}^{2} H_{\beta}^{2}\right)-\operatorname{tr}\left(H_{\alpha} H_{\beta}\right)^{2} & =\frac{1}{2} \sum_{\alpha, \beta} \operatorname{tr}\left(H_{\alpha} H_{\beta}-H_{\beta} H_{\alpha}\right)^{2} \\
& \leq \frac{1}{2} \operatorname{sgn}(p-1)\left(\sum_{\alpha} \operatorname{tr} H_{\alpha}^{2}\right)^{2}
\end{aligned}
$$




$$
=\frac{1}{2} \operatorname{sgn}(p-1) S^{2} .
$$

We also have

$$
\sum_{\alpha, \beta}\left[\operatorname{tr}\left(H_{\alpha} H_{\beta}\right)\right]^{2} \geq \frac{S^{2}}{p} .
$$

Proof of Theorem 1.3. For any $0 \leq a \leq 1$, from (6)-(12) we have

$$
\begin{aligned}
\sum_{i, j, \alpha} h_{i j}^{\alpha} \triangle h_{i j}^{\alpha} \geq & -\frac{8}{3}(1-\delta)(n-1)^{\frac{1}{2}}(p-1) S-n S+(1-a) n \delta S \\
& +(1+a) n K_{\min } S+\left(-\frac{(1-a)}{2} \operatorname{sgn}(p-1)+\frac{a}{p}\right) S^{2} .
\end{aligned}
$$

Take $a=\frac{p}{p+2} \cdot \operatorname{sgn}(p-1)$. From (13) we have

$$
\begin{aligned}
\frac{1}{2} \triangle S= & \sum_{\alpha} \sum_{i, j, k}\left(h_{i j k}^{\alpha}\right)^{2}+\sum_{i, j, \alpha} h_{i j}^{\alpha} \triangle h_{i j}^{\alpha} \\
\geq & \sum_{\alpha} \sum_{i, j, k}\left(h_{i j k}^{\alpha}\right)^{2}-\frac{8}{3}(1-\delta)(n-1)^{\frac{1}{2}}(p-1) S-n S \\
& +\left(1-\frac{p}{p+2} \cdot \operatorname{sgn}(p-1)\right) n \delta S \\
& +\left(1+\frac{p}{p+2} \cdot \operatorname{sgn}(p-1)\right) n K_{\min } S \\
\geq & \sum_{\alpha} \sum_{i, j, k}\left(h_{i j k}^{\alpha}\right)^{2}+n S\left[\left(1+\frac{p}{p+2} \cdot \operatorname{sgn}(p-1)\right) K_{\min }\right. \\
& \left.-\frac{8}{3 n}(1-\delta)(n-1)^{\frac{1}{2}}(p-1)-1+\left(1-\frac{p}{p+2} \cdot \operatorname{sgn}(p-1)\right) \delta\right]
\end{aligned}
$$

It follows from our assumption and the maximum principle that $S$ is a constant. Hence $\sum_{\alpha} \sum_{i, j, k}\left(h_{i j k}^{\alpha}\right)^{2}=0$ and all the inequalities in (7)-(12) are equalities. Let

$$
A=-\sum_{\alpha} \sum_{i, j, k} h_{i j}^{\alpha}\left(\bar{R}_{\alpha k i k j}+\bar{R}_{\alpha i j k k}\right) .
$$

Since (7)-(12) are equalities, from (5) we have

$$
A=-2(1-\delta)(n-1)^{\frac{1}{2}}(p-1) S-n S+n \delta S .
$$

We let

$$
\omega=\sum_{k} \sum_{\alpha} \sum_{i, j}\left(h_{i k}^{\alpha} \bar{R}_{\alpha j i j}+h_{i j}^{\alpha} \bar{R}_{\alpha i j k}\right) \omega_{k} .
$$

Then

$$
\operatorname{div} \omega=\sum_{k} \sum_{\alpha} \sum_{i, j}\left(h_{i k}^{\alpha} \bar{R}_{\alpha j i j}+h_{i j}^{\alpha} \bar{R}_{\alpha i j k}\right)_{k}
$$




$$
\begin{aligned}
& =\sum_{\alpha} \sum_{i, j, k} h_{i j}^{\alpha}\left(\bar{R}_{\alpha k i k j}+\bar{R}_{\alpha i j k k}\right) \\
& =-A .
\end{aligned}
$$

By (16) and (17) we have

$$
\begin{aligned}
0 & =\int_{M}\left[-2(1-\delta)(n-1)^{\frac{1}{2}}(p-1) S-n S+n \delta S\right] d \mu \\
& =\int_{M}\left[-2(n-1)^{\frac{1}{2}}(p-1)-n\right](1-\delta) S d \mu .
\end{aligned}
$$

Since the integrand of (18) is non-positive, we have $(1-\delta) S=0$. Hence $S=0$ or $\delta=1$. If $S=0$, then $M$ is totally geodesic. If $\delta=1$, then $N=S^{n+p}$, and our assumption reduces to $K_{M} \geq \frac{p}{2 p+1} \operatorname{sgn}(p-1)$. From Theorem 1.2 we see that either $M$ is the totally geodesic sphere, the standard immersion of the product of two spheres, or $M$ is the Veronese surface in $S^{4}$. This completes the proof of Theorem 1.3.

\section{References}

[1] S. S. Chern, M. do Carmo, and S. Kobayashi, Minimal submanifolds of a sphere with second fundamental form of constant length, In: Functional analysis and relathed fields, pp. 59-75. Berlin, Heidelberg, New York, Springer, 1970.

[2] Q. Ding and Y. L. Xin, On Chern's problem for rigidity of minimal hypersurfaces in the spheres, Adv. Math. 227 (2011), no. 1, 131-145.

[3] J. Q. Ge and Z. Z. Tang, A proof of the DDVV conjecture and its equality case, Pacific J. Math. 237 (2008), no. 1, 87-95.

[4] S. I. Goldberg, Curvature and Homology, Academic Press, London, 1998.

[5] J. R. Gu and H. W. Xu, On Yau rigidity theorem for minimal submanifolds in spheres, preprint, arxiv:1102.5732v1.

[6] T. Itoh, On Veronese manifolds, J. Math. Soc. Japan 27 (1975), no. 3, 497-506.

[7] _ Addendum to my paper "On Veronese manifolds", J. Math. Soc. Japan 30 (1978), no. 1, 73-74.

[8] B. Lawson, Local rigidity theorems for minimal hypersurfaces, Ann. of Math. (2) 89 (1969), 179-185.

[9] A. M. Li and J. M. Li, An intrinsic rigidity theorem for minimal submanifolds in a sphere, Arch. Math. (Basel) 58 (1992), no. 6, 582-594.

[10] Z. Lu, Proof of the normal scalar curvature conjecture, arXiv:0711.3510v1.

[11] J. Simons, Minimal varieties in Riemannian manifolds, Ann. of Math. (2) 88 (1968), 62-105.

[12] W. D. Song, On minimal submanifolds in a locally symmetric space, Chinese Ann. Math. Ser. A 19 (1998), 693-698.

[13] S. M. Wei and H. W. Xu, Scalar curvature of minimal hypersurfaces in a sphere, Math. Res. Lett. 14 (2007), no. 3, 423-432.

[14] H. W. Xu, On closed minimal submanifolds in pinched Riemannian manifolds, Trans. Amer. Math. Soc. 347 (1995), no. 5, 1743-1751.

[15] H. C. Yang and Q. M. Cheng, Chern's conjecture on minimal hypersurfaces, Math. Z. 227 (1998), no. 3, 377-390.

[16] S. T. Yau, Submanifolds with constant mean curvature I, II, Amer. J. Math. 96, 97 (1974, 1975), 346-366, 76-100. 
Department of Mathematics

ZheJiang Agricultural and Forestry University

ZheJiAng Lin'An 311300, P. R. ChinA

E-mail address: caoshunjuan@126.com 